PROCEEDINGS OF THE

AMERICAN MATHEMATICAL SOCIETY

Volume 129, Number 5, Pages 1335-1348

S 0002-9939(01)05999-8

Article electronically published on January 8, 2001

\title{
TOPOLOGICAL KKM THEOREMS AND GENERALIZED VECTOR EQUILIBRIA ON G-CONVEX SPACES WITH APPLICATIONS
}

\author{
EL MOSTAFA KALMOUN AND HASSAN RIAHI \\ (Communicated by Jonathan M. Borwein)
}

\begin{abstract}
In the present paper, slightly modifying the topological KKM Theorem of Park and Kim (1996), we obtain a new existence theorem for generalized vector equilibrium problems related to an admissible multifunction. We work here under the general framework of G-convex space which does not have any linear structure. Also, we give applications to greatest element, fixed point and vector saddle point problems. The results presented in this paper extend and unify many results in the literature by relaxing the compactness, the closedness and the convexity conditions.
\end{abstract}

\section{INTRODUCTION}

In 1961, Ky Fan [8] extended the classical KKM Theorem to the infinite dimensional case. Later he used this version to state his minimax inequality 9]. Since then, many generalizations have been given by relaxing the compactness, the closedness or the convexity conditions (see for instance [1, 3, 5, 21, 23, 24, 25]). Blum and Oettli in 4] first called these kinds of inequalities "Equilibrium problems" (that is, to find $\bar{x} \in D$ such that $f(\bar{x}, y) \geq 0$ for all $y \in D$, where $D$ is a given set and $f: D \times D \rightarrow \mathbb{R}$ is a given function. The point $\bar{x}$ is then named an equilibria). Then they collected many examples showing the significance of the treatment of this equilibrium problem. Thus it contains special cases for instance optimization, saddle point, Nash equilibrium, complementarity, variational inequality, fixed point and generalized mechanic problems. In recent years, motivated by the earlier work of Giannessi [10] who first introduced vector variational inequalities in a finite dimensional Euclidean space, the study of equilibrium problems has been extended to the case where $f$ takes values in topological vector spaces (we refer for example to Oettli-Schläger [17, Kalmoun-Riahi [13] and the references given there).

Until 1983, all the KKM theorems, the equilibrium problems and the related topics were considered and studied in topological vector spaces in the most general framework. In this setting, convexity assumptions play a crucial role in solving this variety of problems. Horvath [11, 12, replacing convex hulls by contractible subsets, gave a purely topological version of the KKM Theorem. This has motivated other

Received by the editors July 9, 1999.

1991 Mathematics Subject Classification. Primary 49J35, 54H25; Secondary 54Cxx, 52A01.

Key words and phrases. KKM theorem, vector equilibria, G-convex space, generalized Gconvexity, admissible multifunctions, transfer closedness, pseudomonotonicity, greatest element, fixed point, vector saddle point. 
mathematicians to go into the question for generalized KKM theorems and minimax inequalities over topological spaces with no linear structure (see for example [2, 6] 7 (19).

In the present paper, slightly modifying the topological KKM Theorem of Park and Kim 19, we obtain a new existence theorem for generalized vector equilibrium problems, related to an admissible multifunction (Theorem 3.1), under the general framework of G-convex space which does not have any linear structure. Next we give applications to greatest element, fixed point and vector saddle point problems. The results presented in this paper extend and unify many results in the literature by relaxing the compactness, the closedness and the convexity conditions.

The paper is organized as follows. In section 2, we first recall some notions and definitions needed in the sequel. Then, by making use of a result of Park and Kim (Theorem 2.0), we state our generalized topological KKM theorems (Theorems 2.1 and 2.2). Section 3 concerns the study of the existence theory by considering the generalized vector equilibria. Thus the main existence result of this paper (Theorem 3.1) is proved in this section by means of a derivative of Theorem 2.2 (Theorem 2.4). Before that we introduce some new concepts about pseudomonotonicity, Gconvexity and transfer closedness of multivalued bifunctions. Section 4 indicates how the results of this paper may be used to ensure some new existence theorems of particular problems. First Theorem 2.2 is used to get the existence of greatest element of a preference binary relation. Next we apply Theorem 2.4 to state a new fixed point theorem. Finally we use Theorem 3.1 to establish the existence of weak saddle point for a vector valued bifunction.

\section{Generalized KKM Results}

We first recall some basic concepts in order to define the setting of this paper. Further details can be found in [19].

A generalized convex space (G-convex space for short) $(X, D, \Gamma)$ consists of a topological space $X$, a nonempty subset $D$ of $X$, and a map $\Gamma: \mathcal{F}(D) \rightrightarrows X$ with nonempty values and satisfying (1) for each $A, B \in \mathcal{F}(D), A \subset B$ implies $\Gamma(A) \subset \Gamma(B)$, and $(2)$ for each $A \subset \mathcal{F}(D)$ with $|A|=n+1$, there exists a continuous function $\Phi_{A}: \Delta_{n} \rightarrow \Gamma(A)$ such that $J \subset A$ implies $\Phi_{A}\left(\Delta_{J}\right) \subset \Gamma(J)$.

Here and subsequently, $\mathcal{F}(D)$ stands for the set of all finite subsets of $D$, $\Delta_{n} \subset \mathbb{R}^{n+1}$ denotes the $n$-dimensional standard simplex and $\Delta_{J}$ the face of $\Delta_{n}$ corresponding to $J$. To simplify notation we write $\Gamma_{A}$ instead of $\Gamma(A)$.

A subset $C$ of $X$ is called G-convex if $\Gamma_{A} \subset C$ for every finite subset $A$ of $D \cap C$.

Examples of a G-convex space are H-space [12, 2], pseudo convex spaces [11, Lassonde's convex space [15, and convex subsets of a topological vector space.

An admissible class $\Im_{c}^{K}(X, Y)$, where $X, Y$ are two topological spaces, consists of multivalued maps $T$ from $X$ to $Y$ such that, for each one and each compact subset $K$ of $X$, there is a multivalued selection of $T$ in $\Im_{c}(K, Y)$, where $\Im_{c}$ consists of finite composites of maps in $\Im$, and $\Im$ is a class of maps satisfying:

(i) $\Im$ contains the class $\wp$ of single-valued continuous functions,

(ii) each $S \in \Im_{c}$ is u.s.c and compact valued, and

(iii) for any polytope $P$, each $S \in \Im_{c}(P, P)$ has a fixed point.

Examples of this class are given in [19].

A multifunction $F$ from a G-convex space $(X, D, \Gamma)$ to a topological space $Y$ is called G-KKM relatively to a map $T: X \rightrightarrows Y$ (TG-KKM for brevity) if for any 
$A \in \mathcal{F}(D), T\left(\Gamma_{A}\right) \subset \bigcup_{x \in A} F(x)$. We shall only call it weakly $T$ G-KKM relative to $T$ (weakly $T$ G-KKM for brevity) when $T\left(\Gamma_{A}\right) \subset \bigcup_{x \in A} \overline{F(x)}$ for all $A \in \mathcal{F}(D)$.

We now introduce the notion of transfer closedness, following [23. $F$ is said to be transfer closed-valued if, for every $y \in Y$ and every $x \in D$ such that $y \notin F(x)$, there exists $x^{\prime} \in D$ such that $y \notin \overline{F\left(x^{\prime}\right)}$. It will be called transfer compactly closed-valued when the last assertion holds true only on compact subsets of $Y$.

Finally, a subset $B$ of a topological space $Y$ is called compactly closed if, for every compact $C$ of $Y$, the set $B \cap C$ is closed in $C$.

Throughout this paper and unless otherwise stated, $(X, D, \Gamma)$ denotes a G-convex space, $Y$ a Hausdorff space, and $T$ a map belonging to the class $\Im_{c}^{K}(X, Y)$.

The first existing result is due to Park and Kim [19].

Theorem 2.0. Let $F: D \rightrightarrows Y$ be a map such that:

(0.1) for each $x \in D, F(x)$ is compactly closed in $Y$;

(0.2) $F$ is $T G-K K M$;

(0.3) there exists a nonempty compact subset $C$ of $Y$ such that either

(i) $\bigcap_{x \in B} F(x) \subset C$ for some $B \in \mathcal{F}(D)$, or

(ii) for each $A \in \mathcal{F}(D)$, there exists a compact $G$-convex subset $C_{A}$ of $X$ containing $A$ such that $T\left(C_{A}\right) \cap \bigcap_{x \in C_{A} \cap D} F(x) \subset C$.

Then $\bigcap_{x \in D} F(x) \cap \overline{T(X)} \cap C \neq \emptyset$.

Using this result we can state our generalized KKM theorems.

Theorem 2.1. Let $F: D \rightrightarrows Y$ satisfy:

(1.1) $F$ is transfer closed-valued;

(1.2) $F$ is weakly TG-KKM;

(1.3) there exists a nonempty compact subset $C$ of $Y$ such that either

(i) $\bigcap_{x \in B} \overline{F(x)} \subset C$ for some $B \in \mathcal{F}(D)$, or

(ii) for each $A \in \mathcal{F}(D)$, there exists a compact $G$-convex subset $C_{A}$ of $X$ containing $A$ such that $T\left(C_{A}\right) \cap \bigcap_{x \in C_{A} \cap D} \overline{F(x)} \subset C$.

Then $\bigcap_{x \in D} F(x) \cap \overline{T(X)} \cap C \neq \emptyset$.

Proof. First we show that $\bigcap_{x \in D} \overline{F(x)}=\bigcap_{x \in D} F(x)$. On the contrary, if there exists $y \in \overline{F(x)}$ for all $x \in D$ and $y \notin F\left(x^{\prime}\right)$ for some $x^{\prime} \in D$, then, by (1.1) there exists $x^{\prime \prime} \in D$ such that $y \notin \overline{F\left(x^{\prime \prime}\right)}$, which is a contradiction. Second, we set $G(x)=\overline{F(x)}$. Hence $G$ satisfies all conditions of Theorem 2.0. Therefore $\bigcap_{x \in D} F(x) \cap \overline{T(X)} \cap C=\bigcap_{x \in D} \overline{F(x)} \cap \overline{T(X)} \cap C \neq \emptyset$.

Remark 2.1. Example 1 and Example 2 in 23 assert that Theorem 2.1 is not included in Theorem 2.0 .

Remark 2.2. For the moment, Theorem 2.1, as well as Theorem 2.0, generalizes Theorem 3 in 20] and Corollary 3 in [11. Moreover, Theorem 2.1] extends Lemma 2.2 in [6], Theorem 2 in [23] and Lemma 1 in [16]. Finally when $X, Y$ are supposed to be nonempty subsets in a topological vector spaces, it extends the classical Fan Lemma $[8]$ which is an infinite dimensional version of the well-known KKM Theorem [14, 22].

Remark 2.3. 1. Both assumptions (0.1) of Theorem 2.0 and (1.1) of Theorem 2.1] are easily checked when the classical hypothesis of closedness of $F(x)$, for every $x \in D$, is assumed. 
2. As it is mentioned in [19], if $X$ is supposed to be a convex space with $\Gamma_{A}=\operatorname{co} A$, then (i) implies (ii). But generally, (i) does not imply (ii) for G-convex spaces. Besides, assumption (1.3) is satisfied provided $T$ is compact by putting $C=\overline{T(X)}$.

Assumption (1.1) can be relaxed to " $F$ is transfer compactly closed valued". The price paid here is a coerciveness condition a little stronger than (1.3) but always more general than the ones considered in the literature.

Theorem 2.2. Let $F: D \rightrightarrows Y$ a map such that:

(2.1) $F$ is transfer compactly closed-valued;

(2.2) $F$ is weakly $T$ G-KKM;

(2.3) there exists a nonempty compact subset $C$ of $Y$ such that either

(i) $\bigcap_{x \in B} \overline{F(x)} \subset C$ for some $B \in \mathcal{F}(D)$, or

(ii) for each $A \in \mathcal{F}(D)$, there exists a compact $G$-convex subset $C_{A}$ of $X$ containing $A$ such that $\bigcap_{x \in C_{A} \cap D} \overline{F(x)} \subset C$.

Then $\bigcap_{x \in D} F(x) \cap \overline{T(X)} \cap C \neq \emptyset$.

Proof. From (2.1), we have $\bigcap_{x \in D} \overline{F(x) \cap K}=\bigcap_{x \in D} F(x) \cap K$ for every compact subset $K$ in $Y$. Setting $K=\bigcap_{x \in D} \overline{F(x)}$, which is compact by (2.3), we obtain $\bigcap_{x \in D} \overline{F(x)}=\bigcap_{x \in D} F(x)$. The conclusion then follows from Theorem 2.0 .

Here are some results which derive from this theorem.

Theorem 2.3. Let $F: D \rightrightarrows Y$ satisfy (2.1) and (2.3). Suppose that there exists a map $G: X \rightrightarrows Y$ such that:

(3.1) for each $x \in X, T x \subset G x$; and

(3.2) for each $A \in \mathcal{F}(D)$ and each $y \in T\left(\Gamma_{A}\right)$,

$$
A \subset X \backslash \bar{F}^{-1}(y) \Rightarrow \Gamma_{A} \subset X \backslash G^{-1}(y),
$$

where $\bar{F}(x)=\overline{F(x)}$.

Then $\bigcap_{x \in D} F(x) \cap C \cap \overline{T(X)} \neq \emptyset$.

Proof. We have only to show that the multifunction $F$ is weakly TG-KKM. Suppose, contrary to our claim, that there exists an $A \in \mathcal{F}(D)$ such that $T\left(\Gamma_{A}\right) \nsubseteq$ $\bigcup_{x \in A} \overline{F(x)}$. Thus there exists $z \in \Gamma_{A}$ and $y \in T(z)$ such that $y \notin \overline{F(x)}$ for every $x \in A$; that is $A \subset X \backslash \bar{F}^{-1}(y)$. Due to (3.2), we have $\Gamma_{A} \subset X \backslash G^{-1}(y)$. It follows that $z \notin G^{-1}(y)$, and hence that $y \notin G(z)$. This contradicts (3.1) since $y \in T(z)$.

Remark 2.4. Assumption (3.2) holds if, for each $x \in D, G(x) \subset \overline{F(x)}$ and, for each $y \in Y, X \backslash G^{-1}(y)$ is G-convex.

The following result will prove extremely useful in stating the main existence theorem of vector equilibria in Section 2.

Theorem 2.4. Let $F, G: D \rightrightarrows Y$ be two maps. Assume that:

(4.0) $G x \subset \overline{F x}$ for all $x \in D$;

(4.1) $F$ is transfer compactly closed-valued;

(4.2) $G$ is weakly $T$ G-KKM;

(4.3) $G$ satisfy the compactness hypothesis (2.3).

Then $\bigcap_{x \in D} F(x) \cap C \cap \overline{T(X)} \neq \emptyset$. 
Proof. The multifunction $\bar{G}$ verifies all conditions of Theorem 2.2 Hence $\bigcap_{x \in D} \overline{G(x)}$ $\cap C \cap \overline{T(X)} \neq \emptyset$. From (4.0), we obtain $\bigcap_{x \in D} \overline{F(x)} \cap C \cap \overline{T(X)} \neq \emptyset$, and consequently $\bigcap_{x \in D} F(x) \cap C \cap \overline{T(X)} \neq \emptyset$, by $(4.1)$.

Remark 2.5. Using the same argument as in the proof of Theorem 2.3, we can see that assumption (4.2) holds providing, for every $x \in D$ and every $y \in Y$, either

a) $T x \subset \overline{G x}$ and $X \backslash \bar{G}^{-1}(y)$ is G-convex, or

b) $T x \subset G x$ and $A \subset X \backslash \bar{G}^{-1}(y)$ implies $\Gamma_{A} \subset X \backslash G^{-1}(y)$ for all $A \in \mathcal{F}(D)$.

Remark 2.5 shows that Theorem 2.4 extends a result of Horvath 12 , Theorem $2]$.

\section{Generalized vector equilibria}

We start by introducing some notions and definitions used in this section. From now on, $Z$ denotes a topological space, $\varphi$ and $\psi$ two multivalued maps from $D \times Y$ to $Z$, and we will consider $K, L: Y \rightrightarrows Z$ as two ordering multivalued maps.

Definition 3.1. We say that the pair $(\varphi, \psi)$ is $(K, L)$-pseudomonotone (resp. weakly ( $K, L)$-pseudo-monotone) if, for each $x \in D$ and each $y \in Y, \psi(x, y) \nsubseteq L L(y)$ implies $\varphi(x, y) \nsubseteq K(y)$ (resp. $\varphi\left(x, y_{\alpha}\right) \nsubseteq K\left(y_{\alpha}\right)$ for some net $\left(y_{\alpha}\right)$ converging to $y$ in $Y)$.

It is easily seen that the weak $(K, L)$-pseudomonotonicity is more general than the $(K, L)$-pseudomonotonicity. If in addition the classical hypothesis of closedness of the set $\{y \in Y: \varphi(x, y) \nsubseteq K(y)\}$ is supposed hold, then the two definitions are equivalent.

We now give a sharpened vectorial version of the diagonal quasi convexity notion [25] on G-convex spaces.

Definition 3.2. $\psi$ is said to be generalized G-convex (resp. weakly generalized G-convex) in its first argument relative to $T$ and $L$, in short $(T, L)$-generalized Gconvex (resp. weakly $(T, L)$-generalized G-convex) in $x$, if, for any $A$ in $\mathcal{F}(D)$ and any $y$ in $T\left(\Gamma_{A}\right)$, we have $\psi(A, y) \nsubseteq L(y)$ (resp. $\psi\left(A, y_{\alpha}\right) \nsubseteq L\left(y_{\alpha}\right)$ for some net $\left(y_{\alpha}\right)$ converging to $y$ in $Y$ ).

Let us consider the convex and real case, that is when $X=Y=D$ is a nonempty convex subset of a topological vector space, $T=1_{X}, Z=\mathbb{R}$ and $\psi$ is a real valued function. If we set $L:=]-\infty, \gamma[$ (resp. $L:=] \gamma,+\infty[$ ) for some $\gamma \in \overline{\mathbb{R}}$, then the $(T, L)$-generalized G-convexity coincides with the $\gamma$-diagonal quasi convexity (resp. concavity) considered by Zhou and Chen in [25]. However, the weak $(T, L)$ generalized G-convexity, which will be called weak $\gamma$-diagonal quasi convexity (resp. concavity), appears like a generalization of this concept, and consequently of the classical ones such as convexity, quasi convexity and diagonal quasi convexity.

Furthermore, in a special case, the two concepts of generalized G-convexity are equivalent.

Proposition 3.1. If the set $\{y \in Y: \psi(x, y) \nsubseteq L(y)\}$ is closed for each $x \in D$, then the assertion that $\psi$ is $(T, L)$-generalized $G$-convex in $x$ is equivalent to the assertion that $\psi$ is weakly $(T, L)$-generalized $G$-convex in $x$. 
Proof. We only need to prove the reverse assertion. Let us consider $A$ in $\mathcal{F}(D)$ and $y$ in $T\left(\Gamma_{A}\right)$ such that there exists $\left(y_{\alpha}\right)$ in $Y$ converging to $y$ and satisfying $\psi\left(A, y_{\alpha}\right) \nsubseteq$ $L\left(y_{\alpha}\right)$. In this way, there exists $x$ in $A$ such that $\psi\left(x, y_{\alpha}\right) \nsubseteq L\left(y_{\alpha}\right)$, and consequently $\psi(x, y) \nsubseteq L(y)$ thanks to the closedness of the set $\{z \in Y: \psi(x, z) \nsubseteq L(z)\}$. From this we conclude that $\psi(A, y) \nsubseteq L(y)$, which completes the proof.

In general, as we can see in the following example, the class of weakly $(T, L)$ generalized G-convex functions strictly contains $(T, L)$-generalized G-convex ones.

Example 3.1. Let us return to the convex and real case. Define

$$
\psi(x, y)= \begin{cases}\gamma-1 & \text { if } x=y \\ \gamma+1 & \text { otherwise }\end{cases}
$$

for all $(x, y) \in X \times X$. Clearly $\psi$ is weakly $\gamma$-diagonally quasi convex but it's not $\gamma$-diagonally quasi convex (consider only a singleton for the finite subset $A$ ).

We now give some conditions that ensure the weak $(T, L)$-generalized G-convexity.

Definition 3.3. 1. $\psi$ is strongly $(T, L)$-quasi G-convex in $x$ if, for any $A \in \mathcal{F}(D)$ and any $y \in T\left(\Gamma_{A}\right)$, we have

$$
\psi\left(A, y_{\alpha}\right) \subset L\left(y_{\alpha}\right) \text { for all } y_{\alpha} \rightarrow y \text { in } Y \Rightarrow \psi\left(\Gamma_{A}, y_{\alpha}\right) \subset L\left(y_{\alpha}\right) \text { for all } y_{\alpha} \rightarrow y \text { in } Y \text {. }
$$

2. $\psi$ is weakly $(T, L)$-quasi G-convex in $x$ if, in condition 1 , the right-hand side of (1) is replaced by

$$
\psi\left(\Gamma_{A}, y\right) \subset L(y) .
$$

3. $\psi$ is $L$-quasi G-convex in $x$ if, for each $y \in Y$, the set $\{x \in D: \psi(x, y) \subset L(y)\}$ is G-convex.

Remark 3.1. It is easy to check that we have the following implications: $L$-quasi G-convexity $\Rightarrow$ strong $(T, L)$-quasi G-convexity $\Rightarrow$ weak $(T, L)$-quasi G-convexity.

Furthermore, both the weak and the strong $(T, L)$-quasi G-convexity imply the weak $(T, L)$-generalized G-convexity for some particular multivalued maps $T$.

Proposition 3.2. Suppose either

(a) $T x \subset c l_{Y}\{y \in Y: \psi(x, y) \nsubseteq L L(y)\}$ for all $x \in X$ and $\psi$ is strongly $(T, L)$-quasi $G$-convex in $x$, or

(b) $T x \subset\{y \in Y: \psi(x, y) \nsubseteq L(y)\}$ for all $x \in X$ and $\psi$ is weakly $(T, L)$-quasi $G$ convex in $x$.

Then $\psi$ is weakly $(T, L)$-generalized $G$-convex in $x$.

Proof. Suppose, by way of contradiction, that there exists $A \in \mathcal{F}(D), x_{0} \in \Gamma_{A}$ and $y \in T x_{o}$ with $\psi\left(A, y_{\alpha}\right) \subset L\left(y_{\alpha}\right)$ for any net $\left(y_{\alpha}\right)$ converging to $y$ in $Y$. Let us first consider the case when assumption (a) holds. Therefore $\psi\left(\Gamma_{A}, y_{\alpha}\right) \subset L\left(y_{\alpha}\right)$ for all $y_{\alpha} \rightarrow y$ in $Y$, and consequently $\psi\left(x_{0}, y_{\alpha}\right) \subset L\left(y_{\alpha}\right)$ for all $y_{\alpha} \rightarrow y$ in $Y$ since $x_{0} \in \Gamma_{A}$. But this contradicts the fact that there exists a net $\left(z_{\alpha}\right)$ converging to $y$ in $Y$ with $\psi\left(x_{0}, z_{\alpha}\right) \nsubseteq L L\left(z_{\alpha}\right)$, which follows from the first condition of (a).

The same reasoning applies to the case where (b) is satisfied; we shall have, by the weak $(T, L)$-quasi G-convexity, $\psi\left(\Gamma_{A}, y\right) \subset L(y)$, which contradicts our first condition of (b). The same reasoning applies to the case where (b) is satisfied; we shall have, by the weak $(T, L)$-quasi G-convexity, $\psi\left(\Gamma_{A}, y\right) \subset L(y)$, which contradicts our first condition of (b). 
Definition 3.4. 1. We say that $\varphi$ is $K$-transfer continuous in $y$ if, for each $y \in Y$ such that for any $x \in D$ there exists $y_{\alpha} \rightarrow y$ in $Y$ with $\varphi\left(x, y_{\alpha}\right) \nsubseteq K\left(y_{\alpha}\right)$, we have $\varphi(x, y) \nsubseteq K(y)$ for all $x \in D$. Or equivalently, for any $(x, y) \in D \times Y$ with $\varphi(x, y) \subset K(y)$, there exist an element $x^{\prime} \in D$ and an open $V \subset Y$ containing $y$ such that $\varphi\left(x^{\prime}, v\right) \subset K(v)$ for all $z \in V$. We shall call it $K$-transfer compactly continuous when it is $K$-transfer continuous on compact subsets of $Y$.

2. $\varphi$ is said to be transfer upper (resp. lower) semi-continuous in $y$ if, for any $(x, y) \in D \times Y$ and any open $U \subset Z$ with $\varphi(x, y) \subset U$ (resp. $\varphi(x, y) \cap U \neq \emptyset$ ), there exist an element $x^{\prime} \in D$ and an open $V \subset Y$ such that $\varphi\left(x^{\prime}, v\right) \subset U$ (resp. $\varphi\left(x^{\prime}, v\right) \cap U \neq \emptyset$ ) for all $v \in V$. It will be called transfer compactly upper (resp. lower) semi-continuous in $y$ when the last assertion holds on every compact subset of $Y$.

The $K$-transfer (resp. compactly) continuity of $\varphi$ is equivalent to saying that the multivalued mapping $x \rightrightarrows\{y \in Y: \varphi(x, y) \nsubseteq K(y)\}$ is transfer (resp. compactly) closed valued. Furthermore, we have:

Proposition 3.3. If $\varphi$ is transfer (resp. compactly) upper semi-continuous in $y$ with compact values and if $K$ has an open graph, then $\varphi$ is $K$-transfer (resp. compactly) continuous in $y$.

Proof. Let $y \in Y$ such that for any $x \in D$ there is a net $\left(y_{\alpha}\right)$ converging to $y$ in $Y$ with $\varphi\left(x, y_{\alpha}\right) \nsubseteq K\left(y_{\alpha}\right)$. Therefore, for every $x \in D$, there exists $z_{\alpha} \in \varphi\left(x, y_{\alpha}\right)$ with $z_{\alpha} \notin K\left(y_{\alpha}\right)$. Then passing to a subnet, if necessary, we can certainly assume that, for every $x \in D$, there exists $z \in \varphi(x, y)$ such that $z_{\alpha} \rightarrow z$. Otherwise, there exists $x^{\prime} \in D$ such that, for every $z \in \varphi\left(x^{\prime}, y\right)$, there is an open $U_{z} \subset Z$ containing $z$ and satisfying, for every $y_{\alpha}^{\prime} \rightarrow y$ in $Y$ and for every $z_{\alpha}^{\prime} \in \varphi\left(x^{\prime}, y_{\alpha}^{\prime}\right), z_{\alpha}^{\prime} \notin U_{z}$ for $\alpha$ sufficiently large. Since $\varphi\left(x^{\prime}, y\right) \subset \bigcup_{z \in \varphi\left(x^{\prime}, y\right)} U_{z}$ and $\varphi\left(x^{\prime}, y\right)$ is compact, $\varphi\left(x^{\prime}, y\right) \subset U=\bigcup_{i=1}^{i=n} U_{z_{i}}$ for some finite subset $\left\{z_{1}, \ldots, z_{n}\right\}$ in $\varphi\left(x^{\prime}, y\right)$. Hence, there exists $x^{\prime \prime} \in D$ such that, for every $y_{\alpha}^{\prime} \rightarrow y$ in $Y$ and for $\alpha$ large enough, $\varphi\left(x^{\prime \prime}, y_{\alpha}^{\prime}\right) \subset U$ because of the transfer upper semicontinuity of $\varphi$. But, we can also write, for some $y_{\alpha} \rightarrow y$ in $Y$ and some $z_{\alpha} \in \varphi\left(x^{\prime \prime}, y_{\alpha}\right), z_{\alpha} \notin U$ for $\alpha$ large enough. It follows that $\varphi\left(x^{\prime \prime}, y_{\alpha}\right) \nsubseteq U$ for some $y_{\alpha} \rightarrow y$ in $Y$, which is a contradiction.

The same argument is still applicable to show the assertion on compact subsets of $Y$. This completes our proof.

We can now formulate our main existence result.

Theorem 3.1. Suppose that:

(5.0) the pair $(\varphi, \psi)$ is weakly $(K, L)$-pseudomonotone;

(5.1) $\varphi$ is $K$-transfer compactly continuous in $y$;

(5.2) $\psi$ is weakly $(T, L)$-generalized $G$-convex in $x$;

(5.3) there is a nonempty compact subset $C$ in $Y$ such that either

(i) there is a finite subset $B$ in $\mathcal{F}(D)$ such that, for every $y \in Y \backslash C$, there exists $x \in B$ with $y \in$ int $_{Y}\{v \in Y: \psi(x, v) \subset L(v)\}$, or

(ii) for every $A \in \mathcal{F}(D)$ there is a compact $G$-convex $C_{A} \subset X$ containing $A$ such that, for every $y \in Y \backslash C$, there exists $x \in C_{A} \cap D$ with $y \in$ int $_{Y}\{v \in Y: \psi(x, v)$ $\subset L(v)\}$.

Then there exists $\bar{y} \in C \cap \overline{T(X)}$ such that $\varphi(x, \bar{y}) \nsubseteq K(\bar{y})$ for all $x \in D$.

Proof. It's a simple matter to see that all conditions of Theorem 2.4 are fulfilled if we take $F x=\{y \in Y: \varphi(x, y) \nsubseteq K(y)\}$ and $G x=\{y \in Y: \psi(x, y) \nsubseteq L(y)\}$ for all 
$x \in D$. Indeed, (4.0), (4.2) and (4.3) follow from (5.0), (5.2) and (5.3) respectively. To deduce (4.1) from (5.1), let $C$ be a compact subset in $Y$ and let us consider $(x, y) \in D \times C$ with $y \notin F x$. Suppose, contrary to our claim, that $y \in c l_{C} F x^{\prime}$ for all $x^{\prime} \in D$. Therefore, for every $x^{\prime} \in D$, there is a net $\left(y_{\alpha}\right)$ in $C$ converging to $y$ and satisfying $\varphi\left(x^{\prime}, y_{\alpha}\right) \nsubseteq K\left(y_{\alpha}\right)$. It follows that $\varphi\left(x^{\prime}, y\right) \nsubseteq K(y)$ for all $x^{\prime} \in D$ since $\varphi$ is $K$-transfer continuous in $y$ on $C$, and hence that $y \in F x^{\prime}$ for all $x^{\prime} \in D$, a contradiction. Theorem 2.4 now shows that

$$
\bigcap_{x \in D} F(x) \cap C \cap \overline{T(X)} \neq \emptyset,
$$

and this leads precisely to our assertion.

Remark 3.2. According to Proposition 3.3, the theorem is still true if we assume, instead of (5.1), that

$(5.1)^{\prime} \varphi$ is transfer compactly upper semi-continuous in $y$ with compact values and $K$ has an open graph.

Moreover, if $K(x)=K$ for all $x \in D$ and $K$ is an open subset of $Y$, then (5.1) is satisfied if we only suppose that $\varphi$ is transfer compactly upper semi-continuous.

Remark 3.3. 1. If we assume that the set $\{y \in Y: \varphi(x, y) \nsubseteq K(y)\}$ is closed, then assumption (5.1) holds. Besides, (5.0) is reduced to

$(5.0)^{\prime}$ the pair $(\varphi, \psi)$ is $(K, L)$-pseudomonotone.

2. When the set $\{y \in Y: \psi(x, y) \nsubseteq L(y)\}$ is supposed to be closed, assumptions (5.2) and (5.3) will reduce respectively to

$(5.2)^{\prime} \psi$ is $(T, L)$-generalized G-convex in $x$, and

$(5.3)^{\prime}$ there is a nonempty compact subset $C$ in $Y$ such that either

(i) there is a finite subset $B$ in $\mathcal{F}(D)$ such that, for every $y \in Y \backslash C$, there exists $x \in B$ with $\psi(x, y) \subset L(y)$, or

(ii) for every $A \in \mathcal{F}(D)$ there is a compact G-convex $C_{A} \subset X$ containing $A$ such that, for every $y \in Y \backslash C$, there exists $x \in C_{A} \cap D$ with $\psi(x, y) \subset$ $L(y)$.

Remark 3.4. The coerciveness hypothesis (5.3) can be dropped if we assume that either $Y$ is compact, or $D$ is G-convex and compact.

The real and convex version of Theorem 3.1 extends Theorem 2.11 in 25, Theorem 4 in 23. (we take $C_{A}=c o(A \cup R) \cap X$ where $R$ is the convex compact which contains $C$ in (4iii)), Theorem 2 in [3], Theorem 2 in [1], Theorem 5.1 in [1] and Theorem 2.1 in 24].

But when we cannot afford a pseudomonotonicity assumption like (5.0), we can apply Theorem 2.3 to ensure the existence of vector equilibria.

Theorem 3.2. Assume that:

(6.1) $\varphi$ is $K$-transfer compactly continuous in $y$;

(6.2) for each $x \in X$ and each $y \in T x, \psi(x, y) \nsubseteq L(y)$;

(6.3) for each $A \in \mathcal{F}(D)$ and each $y \in T\left(\Gamma_{A}\right)$,

$$
\varphi\left(A, y_{\alpha}\right) \subset K\left(y_{\alpha}\right) \text { for all } y_{\alpha} \rightarrow y \text { in } Y \Rightarrow \psi\left(\Gamma_{A}, y\right) \subset L(y) ;
$$

(6.4) there is a nonempty compact subset $C$ in $Y$ such that either

(i) there is a finite subset $B$ in $\mathcal{F}(D)$ such that, for every $y \in Y \backslash C$, there exists $x \in B$ with $y \in$ int $_{Y}\{v \in Y: \varphi(x, v) \subset K(v)\}$, or 
(ii) for every $A \in \mathcal{F}(D)$ there is a compact $G$-convex $C_{A} \subset X$ containing $A$ such that, for every $y \in Y \backslash C$, there exists $x \in C_{A} \cap D$ with $y \in$ int $_{Y}\{v \in Y: \varphi(x, v)$ $\subset K(v)\}$.

Then the assertion of Theorem 3.1 is still true.

Remark 3.5. This result contains as a special case Corollary 2.5 in [7] which is stated in H-spaces for $T=s \in \wp(X, Y)$ and $\varphi$ and $\psi$ are real valued bifunctions.

We can also relax the coerciveness condition by applying Theorem 2.1 In that case, we need $\varphi$ to be $K$-transfer continuous in $y$ on the whole space $Y$.

Theorem 3.3. Suppose that:

(7.0) the pair $(\varphi, \psi)$ is weakly $(K, L)$-pseudomonotone;

(7.1) $\varphi$ is $K$-transfer continuous in $y$;

(7.2) $\psi$ is weakly $(T, L)$-generalized $G$-convex in $x$;

(7.3) there is a nonempty compact subset $C$ in $Y$ such that either

(i) there is a finite subset $B$ in $\mathcal{F}(D)$ such that, for every $y \in Y \backslash C$, there exists $x \in B$ with $y \in$ int $_{Y}\{v \in Y: \psi(x, v) \subset L(v)\}$, or

(ii) for every $A \in \mathcal{F}(D)$ there is a compact $G$-convex $C_{A} \subset X$ containing $A$ such that, for every $y \in T\left(C_{A}\right) \backslash C$, there exists $x \in C_{A} \cap D$ with $y \in$ int $_{Y}\{v \in Y: \psi(x, v) \subset L(v)\}$.

Then there exists $\bar{y} \in C \cap \overline{T(X)}$ such that $\varphi(x, \bar{y}) \nsubseteq K(\bar{y})$ for all $x \in D$.

A particular case of this theorem is obtained by setting $\varphi(x, y)=\psi(x, y)$ and $K(x)=L(x)$ for all $(x, y) \in D \times Y$, and making use of Remark 3.3. This leads to a slight generalization of Corollary 2 in [16].

Theorem 3.4. Suppose that:

(8.1) for each $x \in D$, the set $\{y \in Y: \varphi(x, y) \nsubseteq K(y)\}$ is closed;

(8.2) for each $y \in Y$, the set $\{x \in D: \varphi(x, y) \subset K(y)\}$ is G-convex;

(8.3) for each $x \in D$ and each $y \in T x, \varphi(x, y) \nsubseteq K(y)$;

(8.4) there is a nonempty compact subset $C$ in $Y$ such that either

(i) there is a finite subset $B$ in $\mathcal{F}(D)$ such that, for every $y \in Y \backslash C$, there exists $x \in B$ with $\varphi(x, y) \subset K(y)$, or

(ii) for every $A \in \mathcal{F}(D)$ there is a compact $G$-convex $C_{A} \subset X$ containing $A$ such that, for every $y \in Y \backslash C$, there exists $x \in C_{A} \cap D$ with $\varphi(x, y) \subset K(y)$.

Then the conclusion of Theorem 3.1 holds true.

We have thus stated Theorem 1.3 in 13 in G-convex spaces under a relaxed compactness condition. The classical Fan minimax inequality 9] can be deduced by setting $D=X=Y, Z=\mathbb{R}, T=i d_{X}, K(x)=\mathbb{R}_{+}^{*}$ and $\varphi(x, y)=f(x, y)-$ $\sup _{x \in X} f(x, x)$ for all $x, y \in X$.

Let us now turn to Theorem 3.1 and mention two important consequences. First, Proposition 3.2 and Theorem 3.1 can be summarized by saying:

Theorem 3.5. Under the assumptions of Theorem 3.1 with (5.2) replaced by (9.2) either

(a) $T x \subset \operatorname{cl}_{Y}\{y \in Y: \psi(x, y) \nsubseteq L(y)\}$ for all $x \in X$ and $\psi$ is strongly $(T, L)$ quasi $G$-convex in $x$, or

(b) $T x \subset\{y \in Y: \psi(x, y) \nsubseteq L(y)\}$ for all $x \in X$ and $\psi$ is weakly $(T, L)$-quasi $G$-convex in $x$.

Then the assertion of Theorem [3.1] remains true. 
Remark 3.6. This result extends Lemma 2 in [16] and Lemma 2.3 in [6] which is given in H-spaces. Furthermore, when $D=X$ and $Y$ are two nonempty subsets of two topological vector spaces, Theorem [3.5 extends a main result of Oettli and Schläger [17, Theorem 1] by relaxing the convexity condition on $\psi$, the continuity hypothesis on $\varphi$ and the pseudomonotonicity assumption on the pair $(\varphi, \psi)$. It also contains as a special case Theorem 1.2 in our previous paper [13].

Next we get the following improvement of Theorem 3 in [17].

Theorem 3.6. Let $X=D=Y$ be a nonempty convex subset of a topological vector space. Suppose that:

(10.0) for every $x, y \in X, \varphi(x, y) \nsubseteq L(x)$ implies $\varphi\left(y, x_{\alpha}\right) \nsubseteq K\left(x_{\alpha}\right)$ for some $x_{\alpha} \rightarrow x$ in $X$;

(10.1) $\varphi$ is K-transfer compactly closed valued;

(10.2) $\varphi\left(x_{\alpha}, x\right) \nsubseteq L\left(x_{\alpha}\right)$ for some $x_{\alpha} \rightarrow x$ in $X$;

(10.3) for each $x \in X$, the set $\{y \in X: \varphi(x, y) \subset L(x)\}$ is convex;

(10.4) for every $x, y \in X,\{u \in] x, y[: \varphi(u, x) \nsubseteq \nsubseteq K(x)\} \cap\{u \in] x, y[: \varphi(u, y) \subset L(x)\}$ $=\emptyset$;

(10.5) for every $x, y \in X$, the set $\{u \in[x, y]: \varphi(u, y) \nsubseteq L(x)\}$ is closed;

(10.6) there is a nonempty compact subset $C$ in $X$ such that, for every $A \in \mathcal{F}(X)$, there is a compact convex $C_{A} \subset X$ containing $A$ and satisfying, for every $x \in Y \backslash C$, that there exists $y \in C_{A}$ with $x \in$ int $_{X}\{u \in X: \varphi(u, y) \subset K(u)\}$.

Then there exists $\bar{x} \in X$ satisfying $\varphi(\bar{x}, y) \nsubseteq L(\bar{x})$ for all $y \in X$.

Proof. We see at once that all the conditions in Theorem 3.5 are satisfied by setting $\psi(x, y)=\varphi(y, x)$ for all $x, y \in X$ and $T=i d_{X}$. Precisely (10.0), (10.1) and (10.6) lead to (5.0), (5.1) and (5.3) respectively, and (8.2) follows from both (10.2) and (10.3). Therefore there exists $\bar{x} \in X$ such that

$$
\varphi(y, \bar{x}) \nsubseteq K(\bar{x}) \text { for all } y \in X .
$$

Now let $y \in X$ and let us set $y_{t}=t y+(1-t) \bar{x}$ for $\left.t \in\right] 0,1[$. Applying (2) we obtain $\varphi\left(y_{t}, \bar{x}\right) \nsubseteq K(\bar{x})$. Thus we must have $\varphi\left(y_{t}, y\right) \nsubseteq L(\bar{x})$, for otherwise assumption (10.4) will be contradicted. From (10.5) it follows that $\varphi(\bar{x}, y) \nsubseteq L L(\bar{x})$, and this is true for any $y \in X$. This proves the theorem.

Remark 3.7. Assumption (10.4) holds if we suppose the following hypotheses:

(i) whenever $x, y \in X$ and $u \in] x, y[$, then $\varphi(u, x) \nsubseteq K(x)$ and $\varphi(u, y) \subset L(x)$ imply $\varphi(u, v) \nsubseteq c l K(x)$ for all $v \in] x, y[$,

(ii) $\varphi(x, x) \subset \operatorname{cl} K(y)$ for all $x, y \in X$.

Moreover, when we let $K(x)=-L(x)=$ int $P$ for all $x \in X$, where $P$ is a closed convex and pointed cone in $X$, conditions (10.2) and (10.3) will be easily checked by (ii) and (i) respectively (see for instance [13, Corollary 1.1]).

\section{Applications}

4.1. Greatest element of a preference relation. Let us consider $D \subset X$ as the set of possible shares among which an economic agent has to make his choice. A binary relation, say $\succeq$, is considered on $X$ and it will be called the preference relation. For $x, y \in X, y \succeq x$ is read $x$ is not preferred to $y$. We will write the negation of $\succeq$ as $\prec$. A point $\bar{y} \in D$ is called greatest element of $\succeq$ on $D$ if $\bar{y} \succeq x$ for all $x \in D$. It's a share that is the most desired in $D$. The existence of such an element is ensured by Theorem 4.1 
Definition 4.1. The preference relation $\succeq$ is said to be transfer compactly upper continuous on $D$ [23] if, for any $(x, y) \in D \times D$ with $y \prec x$, there exists an element $x^{\prime} \in D$ and a compactly open $U_{y} \subset X$ containing $y$ such that $z \prec x$ for all $z \in U_{y} \cap D$.

Definition 4.2. $\succeq$ is said to be weakly generalized G-convex if, for each $A \in \mathcal{F}(D)$ and each $y \in \Gamma_{A}$, there exists $x \in A$ and $y_{\alpha} \rightarrow y$ in $X$ such that $y_{\alpha} \succeq x$.

Theorem 4.1. Assume that:

(1) $\succeq$ is transfer compactly upper continuous on $D$;

(2) $\succeq$ is weakly generalized $G$-convex;

(3) there is a nonempty compact subset $C$ in $D$ such that either

(i) there is a finite subset $B$ in $\mathcal{F}(D)$ such that, for every $y \in X \backslash C$, there exists $x \in B$ with $y \in$ int $_{X}\{v \in X: v \prec x\}$, or

(ii) for every $A \in \mathcal{F}(D)$ there is a compact $G$-convex $C_{A} \subset X$ containing $A$ such that, for every $y \in X \backslash C$, there exists $x \in C_{A} \cap D$ with $y \in$ int $_{X}\{v \in X: v \prec x\}$.

Then there exists $\bar{y} \in C$ greatest element of $\succeq$ on $D$.

Proof. The idea is to take $Y=X, T=i d_{X}$ and $F(x):=\{y \in X: y \succeq x\}$ for all $x \in D$, and then to apply Theorem 2.2

This result improves and extends Theorem 5 in $[23$ to G-convex spaces by relaxing the continuity and the coerciveness conditions.

4.2. Fixed point. We give in this subsection two coincidence results related to admissible multifunctions for multivalued mappings in G-convex spaces.

The first result is a G-convex version of Proposition 3.1 in [11] related to admissible multifunctions.

Proposition 4.1. Let $R: D \rightrightarrows Y$ a map such that $R^{-1}(y)$ is $G$-convex for all $y \in T(X)$ and the multifunction $R^{-}$, given by $R^{-}(x)=Y \backslash R(x)$, is not $T G-K K M$. Then there exists $\bar{x} \in D$ satisfying $T \bar{x} \cap R \bar{x} \neq \emptyset$.

Proof. Since $R^{-}$is not TG-KKM, there exists $A \in \mathcal{F}(D)$ such that $T\left(\Gamma_{A}\right) \nsubseteq$ $\bigcup_{x \in A} R^{-}(x)$. Therefore there exist $\bar{x} \in \Gamma_{A}$ and $\bar{y} \in T \bar{x}$ with $\bar{y} \in R x$ for all $x \in A$. It follows that $x \in R^{-1}(\bar{y})$ for all $x \in A$, and consequently $A \subset R^{-1}(\bar{y})$. However, $R^{-1}(\bar{y})$ is G-convex; hence $\Gamma_{A} \subset R^{-1}(\bar{y})$. We conclude that $\bar{y} \in R \bar{x}$ since $\bar{x} \in \Gamma_{A}$, and the proposition is proved.

Remark 4.1. When we consider the special case, $X=D=Y$ and $T=i d_{X}$, the conclusion of Proposition 4.1 is nothing but the statement that $R$ has a fixed point.

As an application of Theorem 2.2. we give a new fixed point theorem in G-convex spaces.

Theorem 4.2. Let $R: D \rightrightarrows Y$ satisfy:

(1) for each $y \in T(X), R^{-1}(y)$ is $G$-convex;

(2) there is a nonempty compact subset $C$ in $Y$ such that either

(i) there is a finite subset $B$ in $\mathcal{F}(D)$ such that $Y \backslash C \subset \bigcup_{x \in B}$ int $R(x)$, or

(ii) for every $A \in \mathcal{F}(D)$ there is a compact $G$-convex $C_{A} \subset X$ containing $A$ such that $Y \backslash C \subset \bigcup_{x \in C_{A} \cap D} \operatorname{int} R(x)$.

Suppose in addition that there is a transfer compactly open-valued selection $S$ of $R$ satisfying $S^{-1}(y) \neq \emptyset$ for all $y \in C \cap \overline{T(X)}$. Then there exists $\bar{x} \in D$ such that $T \bar{x} \cap R \bar{x} \neq \emptyset$. 
Proof. According to Proposition 4.1, we only need to show that the multifunction $R^{-}$is not TG-KKM. To obtain a contradiction, suppose that this is not true. Then, by applying Theorem 2.4 for $F=S^{-}$and $G=R^{-}$, we have $\bigcap_{x \in D} S^{-}(x) \cap C \cap$ $\overline{T(X)} \neq \emptyset$. Thus there exists $y \in C \cap \overline{T(X)}$ with $x \notin S^{-1}(y)$ for all $x \in D$, which contradicts the fact that $S^{-1}(y) \neq \emptyset$ for all $y \in C \cap \overline{T(X)}$. This completes our proof.

Theorem 4.2 improves Theorem 1 in 3 , Theorem 4.1 in 11 and Theorem 6 in [18].

4.3. Vector saddle point. Now we think of $Z$ as being a topological vector space ordered by a cone $P$ with nonempty interior. Let $f: D \times D \rightarrow Z$ be a single-valued bifunction. We say that $(\bar{x}, \bar{y})$ is a weak saddle point of $f$ if we have

$$
\left\{\begin{array}{l}
f(\bar{x}, y) \notin \text { int } P \text { for all } x \in D \\
f(x, \bar{y}) \notin-i n t P \text { for all } x \in D .
\end{array}\right.
$$

When $Z=\mathbb{R}$ and $P=[0,+\infty[$ then (3) can be written as

$$
\sup _{x \in D} \inf _{y \in D} f(x, y)=\inf _{y \in D} \sup _{x \in D} f(x, y)=0 .
$$

Definition 4.3. We say that $f$ is $P$-transfer lower (resp. upper) semicontinuous in $x$ if, for any $(x, y) \in D \times D$ and for any neighborhood $V$ of $f(x, y)$, there exists a point $y^{\prime} \in D$ and a neighborhood $U_{x}$ of $x$ in $X$ such that $f\left(u, y^{\prime}\right) \in V+P$ (resp. $\left.f\left(u, y^{\prime}\right) \in V-P\right)$ for all $u \in U_{x} \cap D$.

Definition 4.4. $f$ is said to be weakly $P$-generalized G-convex (resp. G-concave) in $y$ if, for each $A \in \mathcal{F}(D)$ and each $x \in \Gamma_{A}$, there exists $y \in A$ such that $f\left(x_{\alpha}, y\right) \notin$ -intP (resp. $f\left(x_{\alpha}, y\right) \notin-i n t P$ ) for some $x_{\alpha} \rightarrow x$ in $D$.

The following theorem is a generalization of Theorem 2.14 in [25]:

Theorem 4.3. Assume that $D$ is a compact subset of $X$. If, in addition,

(1) $f$ is $P$-transfer lower semicontinuous and weakly $P$-generalized $G$-convex in $x$,

(2) $f$ is $P$-transfer upper semicontinuous and weakly $P$-generalized $G$-concave in $y$, then $f$ has a weak saddle point.

Proof. In order to use Theorem 3.1 for $Y=X, T=i d_{X}$ and $K(x)=L(x)=$ int $P$ for all $x \in D$, we have to show that: $f$ is $P$-transfer l.s.c in $x$ (resp. u.s.c in $y$ ) implies that the multivalued mapping $y \in D \rightrightarrows\{x \in D: f(x, y) \notin$ int $P\}$ (resp. $y \in D \rightrightarrows\{x \in D: f(x, y) \notin-i n t P\}$ ) is transfer closed valued. To do this, let $(x, y) \in D \times D$ with $f(x, y) \in \operatorname{int} P$. Therefore int $P$, for it is open, is a neighborhood of $f(x, y)$ in $Z$. By the $P$-transfer lower semicontinuity of $f$ in $x$, there exists $y^{\prime} \in D$ and a neighborhood $U_{x}$ of $x$ in $X$ such that $f\left(z, y^{\prime}\right) \in$ int $P+P$ for all $z \in D \cap U_{x}$. Hence $f\left(z, y^{\prime}\right) \in$ int $P$ for all $z \in D \cap U_{x}$. Thus $x \notin$ $c l_{X}\left\{z \in D: f\left(z, y^{\prime}\right) \notin\right.$ int $\left.P\right\}$, which shows the assertion. By a similar argument, we obtain that $x \in D \rightrightarrows\{y \in D: f(x, y) \notin-i n t P\}$ is transfer closed valued from the $P$-transfer upper semicontinuity of $f$ in $y 1$.

Now we are in position to apply Theorem 3.1. First for $\varphi(x, y)=\psi(x, y)=$ $f(x, y)$ for all $x, y \in D$, we get the existence of $\bar{y} \in D$ satisfying $f(x, \bar{y}) \notin$ int $P$

\footnotetext{
${ }^{1}$ Furthermore, we can show that $f$ is $P$-transfer l.s.c in $x$ (resp. u.s.c in $y$ ) is equivalent to the fact that the multi-application $y \in D \rightrightarrows\{x \in D: f(x, y) \notin \lambda+$ int $P\}$ (resp. $x \in D \rightrightarrows$ $\{y \in D: f(x, y) \notin \lambda-i n t P\})$ is transfer closed valued for all $\lambda \in Z$.
} 
for all $x \in D$. Then for $\varphi(x, y)=\psi(x, y)=-f(y, x)$ for all $x, y \in D$, there exists $\bar{x} \in D$ such that $f(\bar{x}, y) \notin-i n t P$ for all $x \in D$. We conclude that $(\bar{x}, \bar{y})$ is a weak saddle point of $f$, which is our claim.

Remark 4.2. We note that the compactness of $D$ can be relaxed to the coerciveness condition (5.3) applied twice; first to $\psi=f$, then to $\psi(x, y)=f(y, x)$ for all $x, y \in D$. In that case, we can also consider the $P$-transfer l.s.c (resp. u.s.c) of $f$ in $x$ (resp. in $y$ ) only on compact subsets of $D$.

\section{REFERENCES}

[1] G. Allen, Variational inequalities, complementarity problems, and duality theorems, J. Math. Anal. Appl. 58 (1977), 1-10. MR 58:23867

[2] C. Bardaro and R. Ceppitelli, Some further generalizations of KKM theorem and minimax inequalities, J. Math. Anal. Appl. 132 (1988), 484-490 MR 89e:90219

[3] H. Ben-El-Mechaiekh, P. Deguire, and A. Granas, Une alternative non linéaire en analyse convexe at applications, C.R. Acad. Sci. Paris Sér. I Math. 295 (1982), 257-259. MR 84f:52008

[4] E. Blum and W. Oettli, From optimization and variational inequalities to equilibrium problems, Math. Student 63 (1994), 123-145. MR 95i:90089

[5] H. Brézis, L. Nirenberg and G. Stampacchia, A remark on Ky Fan's minimax principle, Boll. Unione Mat. Ital. 4 (1972), 293-300. MR 48:2850

[6] S. S. Chang, B. S. Lee, X. Wu, Y. J. Cho, and G. M. Lee, On the generalized quasi-variational inequality problem, J. Math. Anal. Appl. 203 (1996), 686-711. MR 97i:49017

[7] S. S. Chang and Li Yang, Section theorems on H-spaces with applications, J. Math. Anal. Appl. 179 (1993), 214-231. MR 94i:47098

[8] Ky Fan, A generalization of Tychonoff's fixed point theorem, Math. Ann. 142 (1961), 305310. MR 24:A1120

[9] Ky Fan, A minimax inequality and application, in "Inequalities, III" (Proc. Third Sympos., UCLA, 1969. Dedicated to the Memory of T. S. Motgkin; O. Shisha, Ed.), pp. 103-113, Academic Press, New York, 1972. MR 49:5779

[10] F. Giannessi, Theorems of alternative quadratic programs and complementarity problems, in Variational Inequalities and Complementarity Problems (Cottle, Giannessi and Lions, Eds.), pp. 151-186, John Wiley and Sons, Chicherster, 1980. MR 81j:49021

[11] C. Horvath, Points fixes et cö̈ncidences pour les applications multivoques sans convexité, C.R. Acad. Sci. Paris Sér. I Math. 295 (1983), 403-406. MR 84f:54061

[12] C. Horvath, Some results on multivalued mappings and inequalities without convexity, in "Nonlinear and Convex Analysis-Proceedings in Honor of Ky Fan" (B.-L. Lin and S. Simons, Eds.), pp. 99-106, Dekker, New York, 1987. MR 88g:47019

[13] E. Kalmoun and H. Riahi, Generalized vector equilibrium problems and applications to variational and hemivariational inequalities, Submitted for publication.

[14] B. Knaster, C. Kuratowski, and S. Mazurkiewicz, Ein Beweis des Fixpunktsatsez für ndimensionale simplexe, Fund. Math. XIV (1929), 132-137.

[15] M. Lassonde, On the use of KKM multifunctions in fixed point theory and related topics, J. Math. Anal. Appl. 97 (1983), 151-201. MR 84k:47049

[16] L. J. Lin and S. Park, On some generalized quasi-equilibrium problems, J. Math. Anal. Appl. 224 (1998), 167-181. MR 99f:90182

[17] W. Oettli and D. Schläger, Generalized vectorial equilibria and generalized monotonicity, Func. Anal. Cur. Appl., (M. Brokate and A. H. Siddiki, Eds.), Pitman Res. Notes Math., 377, Longman, London, 1997, pp. 145-154. MR 99b:47106

[18] S. Park, Generalizations of Ky Fan's matching theorems and their applications, J. Math. Anal. Appl. 141 (1989), 164-176. MR 91b:47130

[19] S. Park and H. Kim, Coincidence theorems for admissible multifunctions on generalized convex spaces, J. Math. Anal. Appl. 197 (1996), 173-187. MR 97b:47072

[20] N. Shioji, A further generalization of the Knaster-Kuratowski-Mazurkiewicz theorem, Proc. Amer. Math. Soc. 111 (1991), 187-195. MR 91d:47043

[21] S. Simons, Two-function minimax theorems and variational inequalities for functions on compact and noncompact sets, with some comments on fixed-point theorems, Proc. Symp. Pure Math. 45 (1986), 377-392. MR 87h:49019 
[22] E. Sperner, Neuer Beweis für die Invarianz der Dimensionszahl und des Gebietes, Abh. Math. Sem. Univ. Hamburg 6 (1928), 265-272.

[23] G. Tian, Generalizations of the FKKM theorem and the Ky Fan minimax inequality with applications to maximal elements, price equilibrium, and complementarity, J. Math. Anal. Appl. 170 (1992), 457-471. MR 94b:90014

[24] X. Z. Yuan, The study of minimax inequalities and applications to economies and variational inequalities, Memo. Amer. Math. Soc. 132 (1998), N. 625. MR 98i:49007

[25] J. X. Zhou and G. Chen, Diagonal convexity conditions for problems in convex analysis and quasi-variational inequalities, J. Math. Anal. Appl. 132 (1988), 213-225. MR 89f:90121

Faculté des Sciences I Semlalia, Mathématiques, Université Cadi Ayyad, B.P. 2390, MARRAKECH 40000, MorocCO

E-mail address: ekalmoun@ucam.ac.ma

Faculté des Sciences I Semlalia, Mathématiques, Université Cadi Ayyad, B.P. 2390, MARRAKECH 40000, MorocCO

E-mail address: h-riahi@ucam.ac.ma 